\title{
Anemia Aplástica e Gravidez: Relato de Caso
}

\author{
Aplastic Anemia and Pregnancy: A Case Report
}

\begin{abstract}
Rosiane Alves de Sousa Teles, Danyelle Craveiro de Aquino Veras, Maria de Fátima Veloso Soares, Ana Paula Andrade Augusto, Carlos Augusto Alencar Júnior
\end{abstract}

\section{RESUM0}

A anemia aplástica é distúrbio caracterizado por pancitopenia e medula óssea hipocelular, com substituição gordurosa dos elementos e sem nenhum sinal de transformação maligna ou doença mieloproliferativa. Acomete geralmente adultos jovens e idosos, sem preferência sexual. A maioria dos casos é adquirida, mas pode ocorrer hereditariamente, por distúrbio molecular (anemia de Fanconi). A associação com gravidez é rara, estando relacionada com alta morbidade e mortalidade materna e fetal. Os autores descrevem o caso de uma paciente com anemia aplástica, diagnosticada previamente, cuja gestação complicou com infecção do trato urinário, doença hipertensiva específica da gestação e restrição de crescimento fetal, com parto prematuro eletivo. Apesar das condições adversas na gravidez e parto, mãe $e$ recém-nascido tiveram evolução clínica satisfatória.

PALAVRAS-CHAVE: Anemia aplástica. Gestação de alto risco. Complicações da gravidez. Hipertensão na gestação.

\section{Introdução}

Anemia aplástica é distúrbio da hematopoese caracterizada por marcada redução ou ausência de células eritróides, granulocíticas e megacariocíticas na medula óssea, com resultante pancitopenia. A doença pode ocorrer em qualquer idade, sendo mais comum em adultos jovens (15 a 30 anos) e idosos (acima de 60 anos), incidindo igualmente em homens e mulheres ${ }^{1}$. A maioria dos casos é adquirida, mas pode ocorrer congenitamente, sendo hereditária (anemia de Fanconi) ou não, manifestando-se logo nos primeiros anos de vida. Para as formas adquiridas, uma variedade de fatores causais, incluindo quimioterápicos, toxinas, irradiação e infecções virais têm sido implicados ${ }^{1}$.

Por razões desconhecidas, mulheres podem desenvolver anemia aplástica durante a gravidez, tendo, em geral, resolução espontânea ao término desta, podendo recorrer em novas gestações ${ }^{1-3}$.

Os critérios diagnósticos incluem pancito-

Maternidade Escola Assis Chateaubriand

Serviço de Medicina Materno-Fetal

Departamento de Saúde Materno-Infantil

Universidade Federal do Ceará

Correspondência

Carlos Augusto Alencar Júnior

Rua Vicente Linhares, 1551, apto. 1000 - Aldeota

60135-270 - Fortaleza - Ceará penia, na qual a neutropenia e plaquetopenia podem ser acentuadas, e histopatologia da medula óssea mostrando hipocelularidade com infiltração gordurosa, sem evidência de transformação maligna ou doença mieloproliferativa, sendo os achados semelhantes nas anemias aplásticas congênitas ou adquiridas ${ }^{4}$.

O tratamento sintomático é importante e aumenta a sobrevida dos pacientes. Podem ser usados corticosteróides e andrógenos, sem efeito curativo $^{5}$.

O transplante de medula óssea, única manobra terapêutica corretiva, é o tratamento de escolha para pacientes com menos de 50 anos, com sobrevida de $70-80 \%$. Em pacientes em que não se encontra doador compatível, tem-se como alternativa o tratamento com globulina antitimócito e/ou ciclosporina, com taxa de sucesso de cerca de $50 \%{ }^{4}$.

A gravidez em paciente com anemia aplástica é rara, estando associada a alta morbimortalidade materna e fetal. Quando ocorre, implica mudança na principal proposta terapêutica, visto que o transplante de medula óssea está contra-indicado na gestação.

Os autores descrevem o caso de uma paciente com anemia aplástica diagnosticada antes da gravidez, sua evolução e resultado perinatal. 


\section{Relato do Caso}

CMCM, branca, 21 anos, primigesta, relatava adinamia, cefaléia, náuseas e vômitos desde os vinte anos de idade, quando foi diagnosticada anemia aplástica, por meio de mielograma, que mostrou hipoplasia medular global com aplasia megacariocítica, e estudo histopatológico da medula óssea, que confirmou hipoplasia acentuada da medula hematopoética. Como antecedentes familiares relatava duas irmãs e um primo falecidos e um irmão em tratamento no Hospital Universitário Walter Cantídio (HUWC) pela mesma doença. Em virtude disso, suspeitou-se ser portadora de anemia aplástica congênita (anemia de Fanconi), porém o diagnóstico não foi confirmado. A ausência de alterações ósseas, renais, cardíacas ou hiperpigmentação e manchas café-comleite não parecia sugerir o quadro específico. Havia, ainda, a possibilidade de todos os afetados terem sido comprometidos pelo mesmo fator ambiental, embora o mesmo não tenha sido identificado. Desde então, permaneceu em acompanhamento no Serviço de Hematologia, sem tratamento específico, apenas de suporte.

Iniciou o pré-natal no Serviço de Medicina Materno-Fetal da Maternidade Escola Assis Chateaubriand (MEAC) em maio de 2000, com idade gestacional clínica de 25 semanas e 5 dias, quando foram solicitados os exames de rotina do pré-natal. Nesta ocasião, foi submetida a ultrasonografia, que evidenciou gestação tópica no curso da $24^{\text {a }}$ semana. Apresentava valores hematimétricos extremamente baixos (hematócrito: 8,0\%, hemoglobina: $2,7 \mathrm{~g} / \mathrm{dL}$, leucócitos: 2300/ $\mathrm{mm}^{3}$ e plaquetas: $5000 / \mathrm{mm}^{3}$ ). Compareceu regularmente ao ambulatório de pré-natal de risco elevado, a cada 15 dias em média. $\mathrm{Na} 26^{\mathrm{a}}$ semana apresentou quadro de infecção do trato urinário baixo, sendo internada e tratada com ceftriaxona, $2 \mathrm{~g} /$ dia por 5 dias, e cefalexina, $2 \mathrm{~g} /$ dia por mais 5 dias, recebendo alta hospitalar. $\mathrm{Na} 32^{\mathrm{a}}$ semana, em julho, a ultra-sonografia evidenciou peso fetal abaixo do percentil 10 e dopplervelocimetria da artéria umbilical com aumento da resistência. Foi, então, reinternada na Enfermaria de Patologia Obstétrica da Maternidade Escola Assis Chateaubriand (MEAC) para acompanhamento do bem-estar fetal. Até então, os níveis pressóricos encontravam-se aparentemente normais. Acreditou-se, então, que a restrição do crescimento fetal era decorrente da grave anemia materna.

Evoluiu com aumento dos níveis pressóricos ainda internada e, após cinco dias, foi submetida a nova ultra-sonografia com dopplervelocimetria, na qual foi confirmada a restrição de crescimento e detectada a presença de diástole reversa na artéria umbilical. Por tal razão, e pelo agravamento da doença hipertensiva específica da gravidez, foi indicado parto abdominal, sob anestesia geral. Neste momento, apresentava: hematócrito - 25\%, hemoglobina - 8,4 g/dL, leucócitos - 1500/ $\mathrm{mm}^{3} \mathrm{e}$ plaquetas $-8000 / \mathrm{mm}^{3}$. Recebeu transfusões com concentrado de plaquetas, sendo 5 UI antes do parto abdominal, 5 UI durante e 10 UI após o procedimento.

Apresentou, no pós-operatório imediato, sem sintomatologia prévia, duas crises convulsivas, sendo realizada aplicação de sulfato de magnésio para controle e profilaxia de novas convulsões. Foi transferida para a enfermaria do Serviço de Hematologia do HUWC, no dia seguinte ao parto, onde permaneceu em observação por seis dias. Recebeu alta hospitalar sem seqüelas e com níveis hematimétricos satisfatórios (Ht: 26\%, Hb: 9, 1 $\mathrm{g} / \mathrm{dL}$ e plaquetas: $37.000 / \mathrm{mm}^{3}$ ).

O recém-nascido, sexo masculino, com Apgar 6 e 8 no $1^{\circ}$ e $5^{\circ}$ minuto, respectivamente, foi considerado pequeno para a idade gestacional. Pesou $1.295 \mathrm{~g}$, com estatura de $38 \mathrm{~cm}$ e o Capurro somático foi de 34 semanas e 5 dias. Apresentou desconforto respiratório, permanecendo em UTI neonatal por 27 dias, onde apresentou onfalite e monilíase cutânea, sendo tratado com cefotaxima, amicacina e oxacilina por 14 dias. Foi encaminhado à unidade de médio risco quatro dias após o término da antibioticoterapia, com peso de $1.540 \mathrm{~g}$. Dois dias depois foi matriculado no projeto Canguru, onde permaneceu por onze dias, recebendo alta, após boa evolução clínica, com peso de $1.765 \mathrm{~g}$.

Por desejo materno, houve tentativa de amamentação, que foi contra-indicada, pois a mãe apresentava cansaço e dispnéia quando das mamadas.

\section{Discussão}

O primeiro relato de anemia aplástica na gestação foi efetuado por Ehrlich, em $1888^{4}$. Desde então, poucos casos foram descritos. A rara incidência torna difícil estabelecer uma relação com a gravidez e o manejo ideal nesta situação ${ }^{5}$.

É caracterizada por pancitopenia e medula óssea hipocelular, com substituição gordurosa na histologia, sem evidência de transformação maligna ou doença mieloproliferativa ${ }^{6,7}$.

Várias etiologias têm sido relacionadas; entretanto, em mais da metade dos casos não se encontra uma causa determinada. Nos demais, a doença é provocada principalmente por drogas e 
outros agentes químicos, além de infecções virais, irradiação, leucemias e distúrbios imunológicos ${ }^{1}$.

A anemia aplástica pode ocorrer, também, de forma hereditária (anemia de Fanconi, que é um distúrbio autossômico recessivo). Sua freqüência é estimada em um caso para 360.000 nascimentos $^{8}$. Este número pode estar subestimado, contudo, porque o quadro clínico é muito variável: consiste principalmente de malformações renais e cardíacas, hiperpigmentação, manchas café-comleite e anormalidades ósseas, sobretudo hipoplasia ou ausência dos polegares ou rádios ${ }^{1,8}$. Os pacientes desenvolvem pancitopenia usualmente durante a primeira década de vida e suas complicações são a principal causa de morte. Além disso, portadores de anemia de Fanconi têm maior incidência de malignidade e diabete melito. Cerca de $20 \%$ desenvolverão câncer, principalmente leucemia mielóide, além de outros tumores malignos, entre eles carcinomas epidermóides do trato reprodutivo, pele e sistema gastrintestinal ${ }^{8}$.

A própria gravidez tem sido citada como uma das prováveis causas da anemia aplástica ${ }^{6,7,9}$. Há relatos de mulheres que desenvolveram a doença em gestações consecutivas, com intervalos livres da doença entre las $^{3}$. A patogênese parece ser multifatorial, mas, em geral, o defeito básico, e independente da causa, é uma produção deficiente ou suprimida das células-tronco pluripotentes ${ }^{6,7,9}$. Apesar disso, não há evidências conclusivas que impliquem, com certeza, a gravidez como agente etiológico na patogênese da anemia aplástica ${ }^{4}$.

Os principais riscos para a gestante com anemia aplástica, independente da causa etiológica, são os quadros hemorrágicos e infecciosos, responsáveis por mais de $90 \%$ da mortalidade materna ${ }^{7,9}$.

As pacientes com diagnóstico prévio à gestação parecem ter melhor resultado perinatal e sobrevida do que aquelas diagnosticadas em qualquer época da gravidez ${ }^{7}$.

O tratamento durante a gravidez emprega modalidades terapêuticas diferentes daquelas usadas em mulheres não grávidas ${ }^{7}$. O transplante de medula óssea, que é a terapia de escolha, com sobrevida de 70 a 80\%, está contra-indicado na gestação devido à imunossupressão farmacológica que é necessária antes do procedimento ${ }^{7,10}$. Além disso, hemotransfusões prévias e o próprio estado gravídico aumentam o risco de rejeição ${ }^{9}$.

O uso de andrógenos, que apresentam melhores resultados quando utilizados nas formas mais leves da doença ${ }^{9}$, está contra-indicado em mulheres grávidas de fetos femininos devido à virilização ${ }^{7,9}$. Além disso, toxicidade hepática tem sido uma complicação comum do tratamento com altas doses de andrógenos.
A globulina antitimócito pode ser a melhor opção terapêutica para os casos em que não há doador compativel ou quando o transplante não é possivel. O fator estimulador de colônia, uma glicoproteína que regula a proliferação e a diferenciação das células-tronco hematopoéticas, aumenta a proliferação e migração de neutrófilos. Entretanto, o uso destas terapias durante a gestação ainda necessita de mais estudos ${ }^{6,9,10}$.

O tratamento com altas doses de corticóides pode induzir resposta em uma pequena proporção de pacientes com anemia aplástica, porém, já não são mais utilizados isoladamente devido aos seus efeitos colaterais e a melhores taxas de resposta obtidas com outros agentes ${ }^{1}$.

Qualquer que seja a opção terapêutica, é importante o uso de adequada terapia de suporte. A anemia grave pode levar à restrição de crescimento e morte fetal. A diminuição de plaquetas e leucócitos leva a maior risco de hemorragias e infecções, respectivamente ${ }^{10}$. Recomenda-se, por isso, hemotransfusões para manter a taxa de hemoglobina acima de $8 \mathrm{~g} / \mathrm{dL}$, com o objetivo de melhorar a oxigenação fetal. A transfusão de leucócitos está indicada somente se houver infecção grave $^{10}$. A administração de plaquetas deve ser realizada se a contagem for menor que 20.000/ $\mathrm{mm}^{3}{ }^{7,10}$. Vale destacar que as portadoras de anemia aplástica podem desenvolver aloimunização, especialmente com plaquetas, após repetidas transfusões sangüíneas ${ }^{6}$.

A via de parto preferida é a vaginal, visto que apresenta menor risco de infecção e hemorragia, principalmente se o útero for estimulado a contrair vigorosamente após o parto ${ }^{7,10}$.

Há relatos que indicam resolução da aplasia logo após o parto, não havendo evidências que sugiram que a gestação agrave a anemia aplástica ${ }^{8}$. A antecipação do parto está indicada se o bemestar materno não pode ser mantido com a terapia de suporte ou se a deteriorização do quadro indicar a necessidade urgente do transplante de medula óssea antes do termo ${ }^{7}$.

No caso relatado, a paciente, portadora de anemia aplástica de causa desconhecida, vinha apenas com tratamento de suporte por meio de hemotransfusões antes da gravidez, pois não foi encontrado doador compativel para o transplante de medula óssea, sendo mantida a mesma conduta na gestação.

A gravidez complicou com doença hipertensiva específica da gravidez (DHEG) e restrição do crescimento fetal, fatores responsáveis pela resolução antecipada da gestação. Vale ressaltar não termos encontrado relatos na literatura que mostrem maior incidência de DHEG em pacientes portadoras de anemia aplástica. 
O agravamento da DHEG, culminando em convulsões no puerpério imediato, sem sintomatologia prévia, em paciente com plaquetopenia grave $\left(8000 / \mathrm{mm}^{3}\right)$, aumentou ainda mais o risco materno para sangramento, em especial acidente vascular cerebral hemorrágico.

Apesar das condições adversas da gravidez e do parto, a mãe apresentou evolução clínica satisfatória, recebendo alta 7 dias após o parto. $\mathrm{O}$ recém-nascido apresentou complicações inerentes à prematuridade e à restrição de seu crescimento, sendo liberado 40 dias após seu nascimento, em boas condições.

\section{ABSTRACT}

Aplastic anemia is characterized by a circulating pancytopenia, hypocellularity, and fatty replacement of cellular marrow elements, without evidence of malignant transformation or myeloproliferative disease. It usually affects young and senior adults, without any sexual preference. Most cases of aplastic anemia are acquired, but the disease may also be inherited due to a molecular disorder (Fanconi's anemia). Aplastic anemia in pregnancy is an extremely rare condition with high maternal and fetal morbidity and mortality rates. The authors describe a case of a patient with previously diagnosed aplastic anemia, whose pregnancy was complicated with urinary tract infection, preeclampsia and fetal growth restriction, with elective preterm birth. In spite of the adverse conditions in pregnancy and delivery, mother and newborn had a satisfactory clinical evolution.

KEY WORDS: Aplastic anemia. High risk pregnancy. Pregnancy complication. Hypertension in pregnancy.

\section{Referências}

1. Castro-Malaspira H, O'Reilley RJ. Aplastic anaemia and myelodysplasia. In: Harrison Medicina Interna. $14^{\text {th }}$ ed. New York; Mc Graw Hill; 1998. p.672-8.

2. Fleming AF. Pregnancy and aplastic anaemia. Br J Haematol 1999; 105:315.

3. Bourantas K, Makrydimas G, Georgiou I, Repousis P, Lolis D. Aplastic anaemia: report of a case with recurrent episodes in consecutive pregnancies. J Reprod Med 1997; 42:672-4.

4. Oosterkamp HM, Brand A, Kluin-Nelemans JC, Vandenbroucke JP. Pregnancy and severe aplastic anaemia: causal relation or coincidence? $\mathrm{Br} \mathrm{J}$ Haematol 1998; 103:315-6.

5. Lorenzi TF. Manual de hematologia: propedêutica e clínica. $1^{\text {a }}$ ed. Rio de Janeiro: MEDSI; 1992. Anemias; p.185-277.

6. Ang HY, Ho HK, Linn YC. A case of aplastic anaemia in pregnancy. Aust N Z J Obstet Gynaecol 1999; 39:102-5.

7. Ascarelli MH, Emerson ES, Bigelow CL, Martin JN Jr. Aplastic anemia and immune-mediated thrombocytopenia: concurrent complications encountered in the third trimester of pregnancy. Obstet Gynecol 1998; 91:803-6.

8. Kupfer GM, Naf D, D’Andrea AD. Molecular biology of Fanconi anemia. Hematol Oncol Clin North Am $1997 ; 11: 1045-60$

9. Cunningham FG, Gant NF, Leveno KJ, Gilstrap III LC, Hauth JC, Wenstrom KD. Williams Obstetrics. $21^{\text {th }}$ ed. New York: McGraw Hill; 2001. Hematological disorders; p.1307-38.

10.Ohba T, Yoshimura T, Araki M, et al. Aplastic anemia in pregnancy: treatment with cyclosporine and granulocyte-colony stimulating factor. Acta Obstet Gynecol Scand 1999; 78:458-61.

\section{RBOO}

éumapublicação daFEBRASGO queaceitaartigosprovenientesde ginecologistas, obstetrasedeoutrasespecialidades. Portanto, publique!!! MandejáseuartigoparaRBGO 